\title{
A Proposal for Study of
}

Vacuum Adiabatic Compression of a Relativistic Electron Beam Generated by a Foilless Dlode

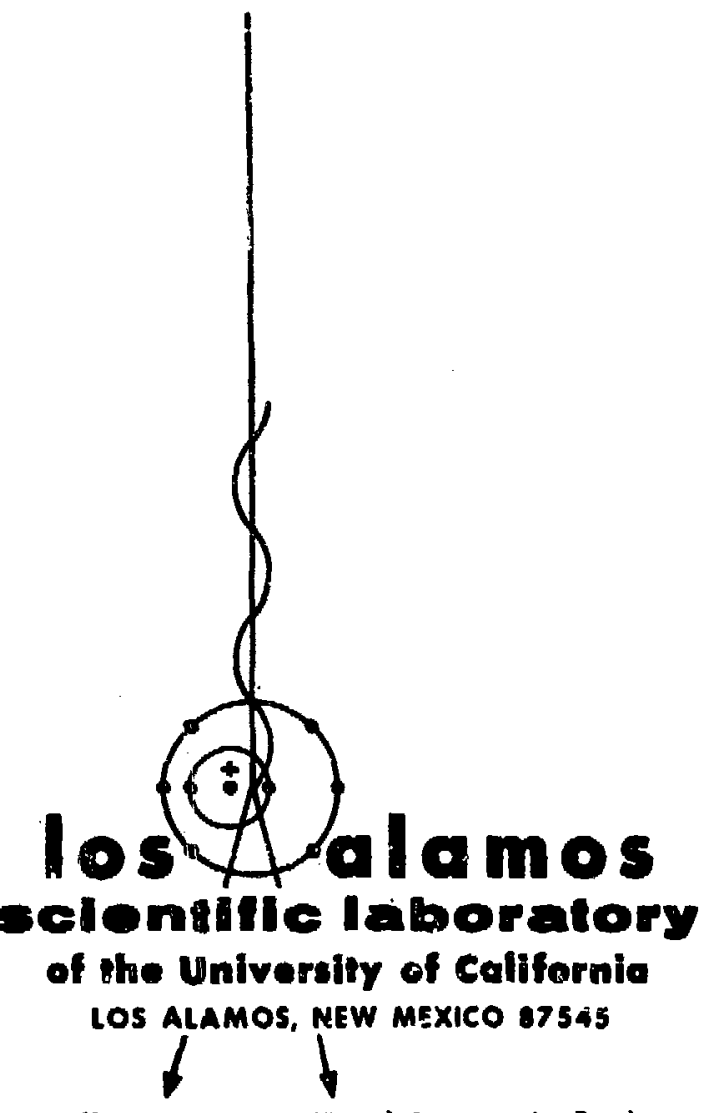

An Affirmative Action/Equal Oppostunily Employer

Lester E. Thode

A solontitie laboratory of the University of Celifornia os ALAMOS, NEW MEXICO 87545 
A PROPOSAL FOR STUDY OF

VACUUM ADIABATIC COMPRESSION OF A RELATIVISTIC ELECTRON BEAM

GENERATED BY A FOILLESS DIODE

by

Lester E. Thode

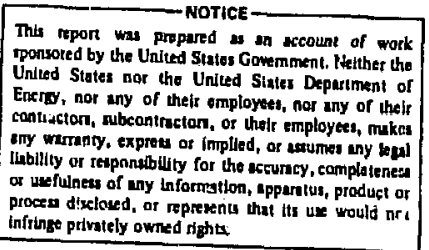

ABSTRACT

A theoretical investigation to study the generation of an intense relativistic electron beam by a foilless diode and subsequent adiabatic compression 18 proposed.

\section{INTRODUCTION}

There are a number of applications for a 5 to 50-MeV electron beam which are of Interest to the Alr Force, Including

(1) submillimeter microwave generaction, ${ }^{1-8}$

(2) Inertially confined plasma $x$-ray source, ${ }^{9-11}$ and

(3) collective ion acceleration. $12-i 8$

In addition to a high beam voltage, these applications ultimately require a beam energy densicy of $10^{19}$ to $10^{22} \mathrm{eV} / \mathrm{cm}^{3}$. This $1 \mathrm{mplies}$ a current density of 50 to $500 \mathrm{kA} / \mathrm{cm}^{2}$, but not necessarily a large net current. Furthermore, the local beam momentum distribution must be sufficiently uniform in energy and well collimated, since the applications of interest depend on the development of largeamplitude, coherent waves. In this context, a measure of the beam quality is the spread in electron velocity components along a beam stream 11ne,

$$
\Delta v_{\|} / c \simeq \theta^{2} / 2+\delta \gamma / \gamma^{3}
$$

where $\theta$ and $\delta \gamma$ are the characteristic angular and energy spread of the local momentum discribution, and $\gamma$ is the relativistic energy factor. The magnitude of 
$\Delta v_{\text {II }}$ which can be tolerated depends upon the wave spectrum associated with the particular application. For example, Inertially confined plasms heating requires

$$
\Delta v_{\|} / c<\gamma^{-i}\left(n_{b} / 2 n_{e}\right)^{1 / 3},
$$

where $n_{b}$ and $n_{e}$ are the beau and plasms electron densities, respectively. High-voltage beam generators exist (Aurora, Pulseiad 1590, and Pulserad 1480), but they are generally operated with a foil diode. A foll diode suffers from shot-to-shot anode foil destruction and assoclated enhanced diode gap closure. ${ }^{19}$ As a result, the initlal dlode gap spacing must be increased, thus lowering the current density by Increasing the beam radius, in opposition to the requirements for the applications of interest. Nevertheless, the foil diode does allow the generation of a beam with a net current greatly exceeding the space charge limiting current, by isolating the vacuum diode region from the plasma or neutral gas region Into which the beam is infected. Th1s configuration is suitable for low-density CTR plasme heating experiments or collective Ion acceleration experiments in which the acceleration is produced by the forma- Ion of a virtual cathode in a space charge 11mited beam. For the most part, past experiments investigating plasma heating ${ }^{20-24}$ and collective ion acceleration $^{12-15}$ have been carried out in this configuration.

Follless diodes offer the potential for overcoming the inherent disadvantages of the anode foll, including the modification of the beam momentum distribution function by classical scattering. In addition, a follless diode is attractive from a repetitive pulsing standpoint. Follless diodes have been used extensively in microwave generation experiments. ${ }^{1-4}$ Beam requirements for inertially confined plasma heating ${ }^{9}$ and collective ion acceleration produced by a train of space charge wells associated with large-amplitude Langmuir ${ }^{18}$ or cyclotron $^{17}$ waves are also consistent with follless diode generation. In the inertially confined plasma heating concept, the beam is propagated in vacuum to the plasma target, passes through solld end plugs, and transfers 30 to 50 percent of 1ts energy to the 30 - to $100-\mathrm{cm}^{3}$ plagna.

Due to the cathode shape and space charge effects, a beam generated by a follless diode is annular with a net current determined by the space charge limit of the vacuum drift tube. For a 5 - to $50-\mathrm{MeV}$ electron beam the space charge limiting current is 100 to $1000 \mathrm{kA}$. Thus, for a small-radius cathode, the beam current density might be limited by the rate at which energy can be delivered 
through the dielectric feed to the diode. In either case, a current density of 10 to $100 \mathrm{kA} / \mathrm{cm}^{2}$ appears realistic for a 5-to $50-\mathrm{MeV}$ follless diode. An alternate approach $1 s$ to use a 3-to 5-MeV follless dlode as an infector for a radial pulse line accelerator. 25,26

To achieve current densities above 10 to $100 \mathrm{kA} / \mathrm{cm}^{2}$, the beam can be adiabatically compressed. To date, three experiments have been performed to investigate the adiabatic compression of a relativistic electron beam. ${ }^{27-29}$ In order to avold the space charge limit on the net beam current, the compression was carried out in the presence of a background gas, and rapld deterioration of beam quality and transmission occurred. As discussed in Ses. Ir, the adlabatic compression. must be performed in vacuum if beam quality is to be preserved, consistent with fotiless diode generation.

Although the approach of using a high-voltage follless dlode to generate an intense electron beam followed by vacuum adiabatic compression, to achieve an energy density of $10^{19}$ to $10^{22} \mathrm{ev} / \mathrm{cm}^{3}$ appears attractive, there is some uncertainty about the quality of the local nomentum distribution function. A theoretical study on the vacuum adlabatic compression of a 5-to 50-MeV electron beam, generated by a foilless diode, is proposed by the Los Alamos Sctentific Latoratory of the Department of Energy. The alm of the study is to determine the feasibility of productng a high-quality beam by this approach. Financial support by the U. S. Air Force Office of Sclentific Research is proposed. The cost of the study is estimated to be $\$ 57,000$ over a one-year period.

To investigate beam generation by follless diode and subsequent vacuum adiabatic compression, the relativistic, electromagnetic particle code CCUBE will be used. The code CCUBE obtains a time-and space-dependent solution in two spatial dimensions for any separable, orthogonal coordinate system. In confunction with the numerical simulations, analytic studies and test particle orbit calculations will be carried out. The electric and mapnetic field configuration for the orbit calculation in the diode will be gulded by the simulation effort.

Results of the study will be reported in the sclentific literature, if approprlate, and also in a document in a form specified by the Air Force office of Scientific Research. It is anticipated that the results of the study will ultimately be incorporated into an experinental investigation on the AFWL Pulserad 1590. 


\section{BACKGROUND}

For sake of discussion, it is assumed that the beam is an infinitely long, unneutralized, constant-density annular column of relativistic electrons. The radial self-electric field is $E_{r}$, and the aximuthal self-magnetic field is $B_{\theta}$. Furtherinore, the beam is immersed in a unfform axial magnetic fleld $B_{z}$, with all the electrons having a radially independent velocity $v_{z}$ in the axial direction. The beam with Inner radius $b$ and outer rsdius $a$ is enclosed in a cylindrical waveguide of radius $R$.

It is well established experimentally that a 1- to 2-MeV electron beam can be generated using a foilless diode. The maximum beam currerit I is determined by the space charge limit of the drift tube through which the beam must propagate, 30,31

$$
I^{S}=17\left(\gamma^{2 / 3}-1\right)^{3 / 2} / G \quad k A \quad \text {, }
$$

where the radial beam geometry factor is

$$
G=1-b^{2} / a^{2}+2 \ln (R / a) .
$$

For these low voltages, the cur ient density in the space charge limited beam is generally less than that due to Child Langmulr limitations associated with the effective diode gap spacing. For a $5-$ to $50-\mathrm{MeV}$ beam, the space charge limit is greatly increased, and for a small-radius cathode the beam current density might be limited by the rate at which energy can be delivered to the diode region through the dielectric feed. In either case, a local current density of 10 to $100 \mathrm{kA} / \mathrm{cm}^{2}$ appears achievable for a 5- to $50-\mathrm{MeV}$ diode. As mentioned previously, an alternate technique is to use a $3-$ to $5-\mathrm{MeV}$ follless diode as an injector for a radial pulse line accelerator. 25,26

To obtain current densities above 10 to $100 \mathrm{kA} / \mathrm{cm}^{2}$, the beam can be adiabdtically compressed. Three experiments have been performed to investigate the adiabatic compression of 1 to $2 \mathrm{MeV}, v / Y=1$ to 10 , electron beams. ${ }^{27-29}$ In these experiments the compression was performed in the presence of a background gas in order to avoid the space charge 1imit on beam propagation. In addition to providing complete space charge neutralization, the background gas also provided significant current neutralization. Rapid current neutralization 
results in a discontinuity in the beam self-magnetic field, ${ }^{32}$ in turn producing a velocity component perpendicular to the external magnetic guide field, in addition to that produced by anode foll scattering. As observed in the three experiments, the combination of self-magnetlc fleld discontinuity and foil scattering leads to a large electron magnctic moment, rapid deterioration of the beam quality and, ultimately, of the Eransmission, even for rather modest compression ratios of $\mathrm{M}=2$ to 4 .

It might be argued that the rather negative experimental results are due to the low diode voltage and insufficient external magnetic field strength. In fact, for compression in the presence of a background gas, the quality and transmission of the center of the beam is primartly limited by collective effects. To examine this point in more detail, the required length of the conpression region can ve compared with the interaction length associated with the two-stream Instability. If the compression is to be adiabatic, the fractional change in the magnetic fleld strength over a Larmor orbit must be small,

$$
\left[\lambda_{c}(z) / B_{z}(z)\right]\left[d B_{z}(z) / d z j \equiv f \ll 1,\right.
$$

where $\lambda_{c}(z)=2 \pi \gamma_{z}(z) / u_{c}(z)$ is the gyro-radius, $B_{z}(z)$ is the component of the external magnetic field in the direction of bean propagation, and $\omega_{c}(z)=|e| B_{z}(z) / m c$. A determination of how small $f$ must be is included in the proposed study. Assuming $f$ to be independent of 2 , expression (5) 1s integrated to give the length of the compression region

$$
\begin{aligned}
z_{c}(\mathrm{~cm}) & =\left[10.6 \cdot \mathrm{fB}_{\mathrm{f}}(\mathrm{kG})\right] \times\left\{\mathrm{M} \sqrt{1-\alpha^{2} / M}-\sqrt{1-\alpha^{2}}\right. \\
& \left.+\alpha^{2} \tanh ^{-1}\left(\sqrt{1-\alpha^{2}}\right)-\alpha^{2} \tanh ^{-1}\left(\sqrt{1-\alpha^{2} / M}\right)\right\}
\end{aligned}
$$

as a function of the compression ratio $M$, maximum perpendicular velocity $\alpha=v_{1}^{f} / c$, and the maximum magnetic fleld strength $B_{f}$. For a high-quality beam $\alpha \ll 1$, and thus

$$
z_{c}(c m)=10.5 \gamma(M-1) / f B_{f}(k G),
$$

which will typically be on the order of a few meters. 
For comparison the nonlinear interaction length assoclated with the twootream instability when $\Delta v_{11} / c \ll \gamma^{-1}\left(n_{b} / 2 n_{e}\right)^{1 / 3}$ is 9

$$
I_{N}=\ln \Lambda\left(n_{e} / n_{b}\right)^{1 / 3} c / \omega_{e}
$$

where $n_{e}$ is the plasma frequency of the background gas and $\Lambda$ is the plasma parameter. In the compression region, $\mathrm{I}_{\mathrm{N}}$ would typicalily be on the order of tens of centimeters, or roughly an order of magnitude shorter than the compression region.

Even if the nonlinear state of the streaming Instablilty is weak, the cransmission of the beam periphery will be limited by the self-magnetic field discontinuity mentioned earlier. A rough rule of thumb for the transverse velocity cumponent due to self-magnetic field discontinuity is that it increases linearly with the bean radius, 32

$$
v_{1} / c \simeq(r / a) \tan ^{-1}\left[B_{\theta}(r=a) / B_{z}\right],
$$

with $B_{\dot{\theta}}(r=a)$ the unneutralized beam self-magnetic field at the beain edge. As the compression ratio is increased, the characteristic magnetic moment at the entrance of the compression region is increased, since the maximum magnetic field strength at the end of the compression region is 1imited by strength of materials considerations. (An exception to this limit might be provided by an explosive-driven flux compressor.) Thus, the presence of the background gas is the limiting factor in obtaining an intense high-quelity beam. It follows that the adiabatic compression must: be performed in vacuum, vich implies a highvoltage diode to increase the space charge 1imit.

In vacuum there are a number of conditions which must be satisfied for beam equilibrium and stability in the compression reglon. ${ }^{33,34}$ In the rest frame of the beam, $B_{z}$ must exceed $E_{r}$;

$$
w_{1}^{2}\left(1-b^{2} / a^{2}\right)<\omega_{c} c / a,
$$

where $\omega_{\perp}^{2}=4 \pi n_{b} e^{2} / \mathrm{m} \gamma$ and $\omega_{c}=|e| B_{z} / m c$. In addition, the centrifugal force 
associated with the $\vec{E} \times \vec{B}$ rotation plus the radial self-field force $E_{r}-v_{z} B_{\theta}$ must be less than the $v_{\theta} B_{z}$ restoring force;

$$
w_{1}^{2}\left(1-b^{2} / a^{2}\right)<0.5 \omega_{c}^{2},
$$

where $v_{\theta}=r \omega_{\theta}(r)$ and $\omega_{\theta}(r)=0.5 \gamma^{-1} \omega_{1}^{2}\left(1-b^{2} / r^{2}\right) \omega_{c}$.

In obtaining expressions (10) and (11), the beam diamagnetic field due to the $\vec{E} \times \vec{B}$ rotation has been neglected. The neglect of the diamagnetic field $B_{Z}^{S}$ Is valid if

$$
\mathrm{B}_{\mathrm{z}}^{\mathrm{S}} / \mathrm{B}_{\mathrm{Z}} \simeq\left[0.5\left(\omega_{1} / \omega_{c}\right)\left(\mathrm{a} \omega_{1} / c\right)\left(1-\mathrm{b}^{2} / \mathrm{a}^{2}\right)\right]^{2} \ll 1
$$

Expression (12) is also related to the condition that the relativistic factor $\gamma=\gamma_{0}\left(1-\beta_{\theta} \gamma_{0}^{2}\right)^{-1 / 2}$ is approximately independent of the beam radius and thus represents a beam quality condition. The radially independent relativistic factor is $\gamma_{0}=\left(1-\beta_{z}^{2}\right)^{-1 / 2}$ and $\beta_{\theta} \gamma$ is given by the term in brackets in Eq. (12).

In the high-voltage regime, the equilibrlum conditions can be satisfled within strength of materials limitations on the external magnetic fleld. For example, if $Y_{0}=60, \mathrm{n}_{\mathrm{b}}=10^{14} \mathrm{~cm}^{-3}, a=1 \mathrm{~cm}$, and $\mathrm{B}_{\mathrm{z}}=200 \mathrm{kG}$, the most stringent condition (12) yields $\beta_{\theta} \gamma_{0}=2 \times 10^{-\overline{2}}$. Also, if the compression is adlabatic, Eqs. (10) through (12) remain constant throughout the compression region.

The preceeding annular beam equilibrium is rot stable. Due to the presence of a shear in angular velocity $v_{\theta}(r)=r \omega_{\theta}(r)$ for $b \neq 0$, flute perturbations $\exp (\operatorname{im} \theta)$ on the beam surface are found to be unstable. The growth rate of this so-called Diocotron Instability is ${ }^{34}$

$$
\delta \simeq 0.5 \gamma^{-1}\left(\omega_{1}^{2} / \omega_{c}\right) P_{m}(b / R, a / R) \quad,
$$

where $P_{m}$ is a geometric factor depending on the beam profile. Thick annular beams are only unstable to the $m=2$ mode, with $P_{\mathrm{Il}}^{\max } \simeq 0.2$. For $(a-b) / R \ll 1$, the higher modes are also unstable with $\mathrm{P}_{\mathrm{m}>>1}^{\max } \simeq 0(1)$. Compraring the instability e-folding time $t \simeq 1 / \delta \simeq 10^{-7}$ to $10^{-6} \mathrm{sec}$ with the characteristic transit time for: beam compression $t=z_{c} / c \simeq 10^{-8} \mathrm{sec}$, the instability hals a negligible effect 
on the beam profile. On a longer time scale, such as needed for collective ion acceleration, the instabllity may have sufficient time to slightly modify the beam profile, however.

Due to the time-dependent, relativistic character of the electron dynamics, the primary technique to be used in the investigation will be numerical simulation. Specifically, the relativistic, electromagnetic particle code CCUBE w111 be used. 35 The code CCUBE obtains a time-and space-dependent solution in two dimensions for any separable, orthogonal coordinate system. The local field strength in each cell can be specified, if desired. Thus, it is possible to have a boundary which is perlodic or irregular, with a flxed or floating potentlal.

A large number of specialized time-integrated numerical measurements such as beam inductance, local electric and magnetic field probes, diamagnetic 1oops, Faraday cups, and calorimeters are implemented in the code. In addition, local beam distribution functions and particle density profiles in both dimensions are avallable.

The code has been successfully used to study space charge limited flow, 36 collective ion acceleration, 37 and beam injection into a plasma with subsequent heating due to the streaming instabilities. 11 To date, the distribution funct:Lon at injection has been assumed to be of the form

$$
f(\theta, \gamma, r) \propto \delta\left(\gamma-\gamma_{0}\right) \exp \left[-\left(\theta-\theta_{r}\right)^{2} / \theta_{s}^{2}\right],
$$

where $\theta_{s}$ is the anode foil scattering angle, $\theta_{r}=r B_{\theta}(r=a) / a B_{z}$, and a is the beam radius. The only code development required to carry out the proposed study is the Implementation of an electron emission ${ }^{38,39}$ algorithm in CCUBE.

The simulations will provide information on the electric and magnetic field configuration in the diode for a limfted number of parameter variations. Using these flelds, test particle orbit calculations can be carried out to extend the parameter variation. In addition, the test particle orbit calculations will provide additional detall of the local beam distcibution function, such as local Larmor radius and temperature. From the simulations, the local distribution functions will be coarse grain in character, typically 100-150 particles $(3 \times 3$ or $3 \times 4$ cells). It 1 s expected that five to ten local distributions across the beam profile can be obtained. Comparison between summed test particle orbits 
and the coarse-grain simulation distribution will provide the check on the test particie technique.

\section{OBJECTIVE}

The investigation of the foilless diode generation of a 5- to 50-MeV electron beam and the vacuum adiabatic compression of such a beam is proposed. The objective is to determine the feasibility of obtaining a beam with a local energy density of $10^{1.9}$ to $10^{22} \mathrm{ev} / \mathrm{cm}^{3}$, with sufficient microscopic quality to be applicable to
(1) submillimeter microwave generation, ${ }^{1-9}$
(2) Inertially confined plasma $x$-ray source, $9-11$ and
(3) collective ton acceleration. ${ }^{12-18}$

IV. PROPOSED RESEARCH PROGRAM

The proposed program is not intended to be a detailed design effort. Rather, a number of features of high-voltage follless diode operation and vacuum adiabatic compression w1ll be clarified, which can be ultimately tncorporated into an experimental study. The investigation is split into two parts. A. Follles, Diode in Cylindrical Coordinates

The first part of the study considers the basic aspects of a simple follless diode in cylindrical coordinates, assuming spatial azimuthal symnetry. The proposed geometry of the diode is shown in Fig.. 1. Four parameters are expected to

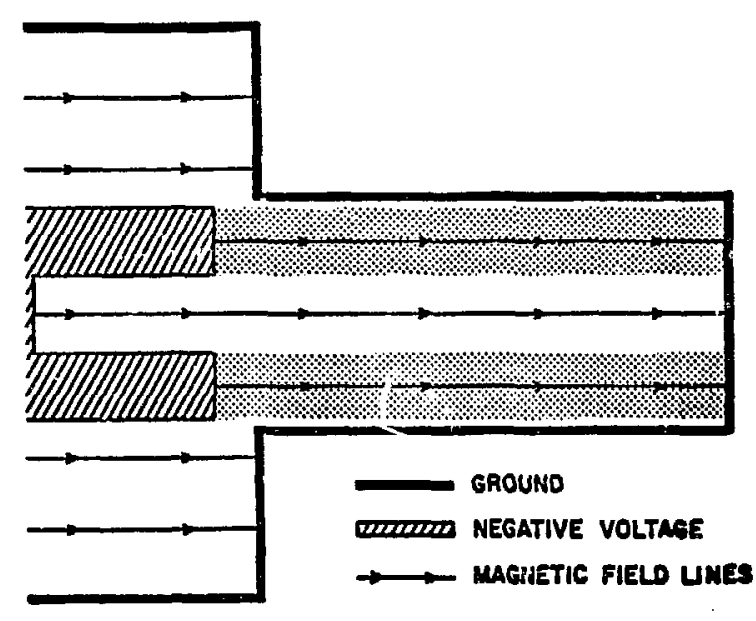

Fig. 1. Proposed geometry for part A (Cylindrical Coordinates). be important in determining the bean quality and intensity:

(1) the relativistic factor

$$
\gamma=\left(1-\beta_{z}^{2}-\beta_{\theta}^{2}\right)^{-1 / 2},
$$

(2) the effective diode gap spacing d through the ratio

$$
I(d) / I^{s},
$$

(3) the diamagnetic and racilally dependent parameter 


$$
\beta_{\theta} \gamma_{0}=0.5\left(\omega_{1} / \omega_{c}\right)\left(a \omega_{1} / c\right)\left(1-b^{2} / a^{2}\right),
$$

and (4) the geometry factor

$$
G=1-b^{2} / a^{2}+2 \ln (R / a)
$$

It is planned to obtain a consistent set of simulations, which contain significant changes in beam quality as $\gamma, I / I^{S}, \beta_{\theta} \gamma$, and $G$ are varied. It is not proposed to obtain simulations involving all combinations of the parameters. For example, since $\beta_{\theta} \gamma_{0} \ll I$ can always be satisfied physically, the effect of Increasing this parameter can be limited to the high-voltage case with only variations in $I / I^{S}$. Also, extensive variation in the geometry factor $G$, which depends primarily on the ratio $b / a$ since $(R-a) / r \ll 1$, is not anticipated. Finaily, a sma?l number of simulations to investigate the effect of the emission temperature are planned.

To provide additional insight into the sensitivity of the beam quality on the various parameters, test particle orbit calculations will be carried out in conjunction with the simulations. In turn, the electric and magnetic field configuration for the test particle calculations will be guided by the simulations.

B. Foilless Diode and Vacuum Adiabatic Compression in Spherical Coordinates

The second part of the study considers the combination of high-voltage foilless operation and adiabatic compression. In addition to the four parameters associated with the diode, the effect of the fractional change in the compression ratio must be considered. If the beam quality is preserved during the adiabatic compression, the parameters $I / I^{S}, G$, atd $\beta_{\theta} \gamma_{0}$ determined at the diode remain constant. The parameters chosen for the simulattoñs will depend to a great extent on the results of the cylindrical diode studies, part A. The proposed geometry

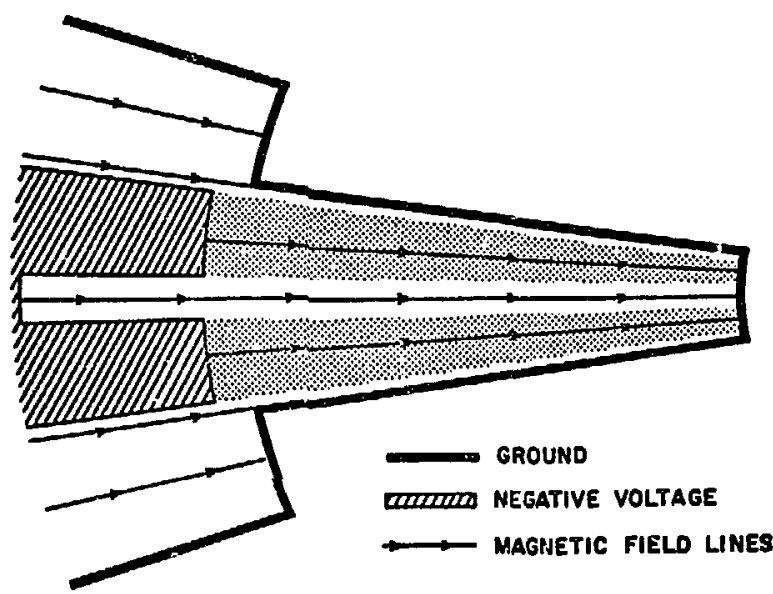

Fig. 2. Proposed geometry for part B (Spherical Coordinates). for part $B$ is shown in Fig. 2. 10 
A small number of simulations are planned to consider the magnitude of $f$, which can be tolerated for high-voltage beam compression. The primary aim of these simulations is to determine a practical upper bound for $f$.

It is expected that the primary effort w11l be to determine the trade-off in beam quality between diode current density and the compression ratio, for a fixed-beam energy density at the extt of the compression region.

V. WORK STATEMENT

Implement an electron emission alogrithm in the two-dimensional particle code CCUBE.

Study the basic aspects of high-and low-voltage follless diode operation in cylindrical coordinates using CCUBE. Obtain a consistent set of simulations which contain significant changes in the beam quality as a function of the 1) ratio of the beam current to the space charge limiting current, 2) dlamagnetic factor, and 3) geometry fastor. It is not proposed to obtain simulations involving all combinations of the preceding parameters.

Solve the relativistic equations of motion using an electric and magnetic field configuration guided by the simulation effort.

Study the basic aspects of high-voltage follless diode operation and vacuum adiabatic compression in spherical coordinates using CCUBE. Determine an upper bound on the fractional change in the external magnetic field strength over a Larmor orbit to insure adiabatic compression. Determine the trade-off In beam quality between current density at the diode and the adiabatic compression ratio for a fixed-beam energy density at the exit of the compression region.

\section{TERM}

The work will be initiated on October 1, 1977, or upon receipt of authorization from the Department of Energy to proceed, whichever is later. The duration of the proposed study is one year. If authorization is not recelved by January 1, 1978, it may become necessary to modify this proposal. 
VII. REPORTING

Results of the study will be reported as required by the U. S. Alr Force office of Scientific Research. If appropilate, the results of the study wili also be reported in the acientific literature.

\section{PERSONREL}

L. E. Thode of Group T-15 (LASL) will be the manager of the proposed study. He will have the general responsibility for the performance and reporting of the proposed work, and for llaison with the Alr Force representatives as required. A major portion of the work will be performed by a LASL postdoctoral fellow under Thade's direction.

In addition to Thode, B. Godfrey, F. Faehl, B. Newberger, and R. Shanahan of Group T-15 (LASL) will act as technical consultants for the proposed study.

IX. cosrs

The cosis to the Department of Energy for the performance of the proposed study are itemized in the following table.

\section{TABLE I}

COST FOK PROPOSED RESEARCH PROGRAM

Proposed starting date: October 1, 1977

Proposed ending date : October 1, 1978

\section{Man-years}

Scientific

Other

\section{Costs}

Salaries

Indirect Costs

Materials and Services

Computer Services

Toma1
$\$ 1000$

13000

4000

19000

$\$ 57000$ 
1. M. Friedman and M. Herndon, "Mlcrowave EmIssion Produced by the Interaction of an Intense Relativistic Ejectron Beam with a Spatially Modulated Magnetic Fleld," Phys. Rev. Lett. 28, 210 (1971).

2. M. Frledman, D. A. Hammer, W. M. Manhelmer, and P. Sprangle, "Enhanced Microwave Emission Due to the Transverse Energy of a Relativistic Electron Beam," Phys. Reэ. Lett. 31, 752 (19?3).

3. M. Freldman and M. Herndon, "Emlssion of Coherent Microwave Radiation from a Relativistic Electron Beam Propagating in a Spatially Modulated Field," Phys. Fluids 16, 1982 (1973).

4. V. L. Granatsteln, M. Herndon, P. Sprangle, Y. Carmel, and J. A. Nation, "Gigawatt Microwave Emission from an Intense Relativistlc Electron Beam," Plasma Phys. 17, 23 (1975).

5. W. M. Manheimer and E. Ott, "Theory of Microwave Generation by an Intense Relativistic Beam in a Rippled Magnetic Fiald," Phys. Fluids 17, 463 (1974).

6. V. L. Granasieln, P. Sprangle, M. Herndon, R. K. Parker, and S. P. Schlesinger, "Microwave Amplification with an Intense Relativistic Electron Beam," J. App1. Phys. 46, 3800 (1575).

7. E. Ott and W. M. Manheimer, "Theory of Microwave Emission by Velocity Space Instabilitles of an Intense Relativistic Electron Beam," IEEE Trans. Plasma Sci. PS-3, 1 (1975).

8. P. Sprangle, R. K. Parker, J. Pasour, M. Herndon, S. P. Schlesinger, and J. L. Seftor, "Realization of a Relativistlc Mirror: Electromagnetic Backscattering from the Front of a Magnetized Relativistic Electron Beam," Phys. Rev. Al4, 1194 (1976).

9. L. E. Thode, "Preliminary Investigation of Anomalous Relativistic Electron Beam Deposition Into Dense ( $10^{17}$ to $10^{20} \mathrm{~cm}^{-3}$ ) Plasma," unpublished.

10. L. E. Thode, "Effect of Electron-Ion Collisions on the Nonlinear State of the Relativistic Two-Stream Instability," Phys. Flu1ds 20, 2121 (1977).

11. L. E. Thode, "Preliminary Investigation of inomalous Relativistic Electron Beam Deposition into Dense $\left(10^{17}-10^{20} \mathrm{~cm}^{-3}\right)$ Plasma," In the Conference Record of the IEEE Fifth International Conference on Plasma Science (Troy, New York, 1977), P. 33 .

12. C. L. Olson, "Theory of Ion Acceleration by Drifting Intense Relativistic Electron Beams. II. Comparison with Experiments," Phys. Fluids 18, 598 (1975); see references.

13. B. Ecker, S. Putnam, and D. Drickey, "Further Studies of Collective Acceleration of Positive Particles Using Intense Electron Beams," IEEE Trans. Nucl. Sc1. NS-20, 305 (1973). 
14. G. W. Kuswa, L. P. Bradley, and G. Yonas, "Ion Acceleration In Electron Beams," IEEE Trans. Nuc1. Sc£. NS-20, 305 (1973).

15. D. C. Straw and p. B. MIller, "Dependence of Ion Acceleration on LImiting Current in Relativistic Electron Beams," Appl. Phys. Lett. 25, 379 (1974).

16. C. L. 0lson, "Theory of Ion Azceleration by Drifting Intense Relativistic Electron Beams; I. Theory," Phys. Flulds 18, 585 (1975).

17. M. L. Sloan and W. E. Drummond, "Autoresonant Accelerator Concept," Phys. Rev. Lett. 31, 1234 (1973).

18. P. Sprangle, A. T. Drobot, and W. M. Manheimer, "Collective Ion Acceleration In a Converging Wavegulde," Phys. Rev. Lett. 36, 1180 (1976).

19. D. R. Dakin and J. Benford, "Closure Velocitles and Blpolar Flow for Intense REB Diodes in $B_{2}$ Fie.2ds," In the Conference Record of the IEEE Fifth International Conference on Plasma Sclence (Troy, New York, 1.977), p. 78.

20. L. E. Thode, "Plasma Heating by Scattered Relativistic Electron Beams: Correlations Among Experiment, Simulation, and Theory," Phys. Flulds 19, 831 (1976); see references for preionized target gas experiments prior to 1975.

21. D. Prono, B. Ecker, N. Bergstron, and J. Benford, "Plasma-Return-Current Heating by Relativistic Elect on Beams with $v / \gamma \sim 10, "$ Phys. Rev. Lett. 35, 438 (1975).

22. R. Okamura, Y. Nakamura, and N. Rawashima, "Microwave Emission from the Magnetized Plasma Heated by a Short Pulse Width Relativistic Electron Beam," Institute of Space and Aeronautical Science Research Note 2 (1975), University of Tokyo, Meguro, Tokyo.

23. A. K. L. Dymoke-Bradshaw, A. E. Dangor, and J. D. Kilkenny, "Plasma Heating by an Intense Relativistic Electron Beam," 6th International Conference on Plasma Physics and Controlled Nuclear Fusion Research (Berchtesgaden, 1976), paper 62-4.

24. B. Juigens, H. J. Hopman, P. de Haan, P. C. de Jagher, and J. Kistemaker, "Energy Transfer of a Relativistic Electron Beam to a Plasma," 6th International Conference on Plasma Physics and Controlled Nuclear Fusion Research (Berchtesgaden, 1976), paper G2-1.

25. A. I. Pavlovsk11, V. S. Bosamykin, G. D. Kuleshov, A. I. Gerastmov, V. A. Tananakin, and A. P. Klement'ev, "Multlelement Accelerators Based on Radial Lines," Dokl. Akad. Nauk SSSR 222, 817 (1975) [Sov. Phys. Dok1. 20, 441 (1975)].

26. V. I. Kazacha and I. V. Kozhukhov, "Use of Radial Transmission Lines in Pulsed Accelerators," Zh. Tekh. Fiz. 46, 1477 (1976) [Sov. Phys. Tech. Phys. 21, 841 (1976)]. 
27. P. E. Bolduc and E. I. Patterson, "Magnetic Focusing of a Rèlativistic Electron Beam: Experiment," J. Appl. Phys. 43, 4006 (1972).

28. H. Davitian and J. Nation, "Compression of Intense Relativistic Electron Beams," Laboratory of Plasma Studies Report 非134, Corne11 Iniversity (1973).

29. C. Stallings, J. Benford, and K. Childers, "Magnetic Focusing of Intense Relativistic Electron Beams," Plasma Phys. 18, 317 (1976).

30. E. Ott, T. M. Antonsen, and R. V. Lovelace, "Theory of Follless Diode Generation of Intense Relativistic Electron Beams," Phys. Flu1ds 20, 1180 (1977).

31. M. E. Read and J. A. Nation, "Space-Charge Limits In Unneutralized RelativIstic Electron Beams," J. Plasma Phys. 13, 127 (1975).

32. T. S. T. Young and P. Spence, "Model of Magnetic Compression of Re1ativistic Electron Beams," App1. Phys. Lett. 29, 465 (1976).

33. B. N. Brejzman and D. D. Ryutov, "Powerful Relativistic Electron Beams in a Plasma and In a Vacuum (Theory)," Nuslear Fuston 14, 873 (1974).

34. R. C. Davidison, Theory of Nonneutral Plasmas (Benjamin, Massachusetts, 1974).

35. The code was designed and implementea by B. B. Godfrey, see the "Theoretical DIvision Annual Report - FY76," LA-6816-PR (1976), p. 148.

36. L. E. Thode, B. B. Godfrey, and W. R. Shanahan, "Vacuum Propagation of Solid Relativistic Electron Beams: Correlations Among Experiment, Simulation, and Theory," submitted to Phys, Flutds.

37. B. B. Godfrey, "Numerical Simulation of sutoresonant Ion Acceleration," IEEE Trans. Plasma Sci. PS-5, 223 (1977).

38. R. Lee and S. A. Goldstein, "Computer Simulations of Time Dependent Ion and Pinched Electron Flow in Relativistic Diodes," in the Conference Record of the IEEE Fifth International Conference on Plasma Science (Troy, New York, 1977), p. 78 .

39. J. W. Poukey, "Computer Sirulations of Relativistic E1ectron Beam D1odes," in the Conference Record of the IEEE Fifth International Conference on Plasma Science (Troy, New York, 1977), p. 79. 